\title{
Effectiveness of Micronutrient Powders (MNP) in women and children
}

Rehana A Salam¹, Ceilidh MacPhail², Jai K Das', Zulfiqar A Bhutta ${ }^{1,3^{*}}$

\begin{abstract}
Introduction: More than 3.5 million women and children under five die each year in poor countries due to underlying undernutrition. Many of these are associated with concomitant micronutrient deficiencies. In the last decade point of use or home fortification has emerged to tackle the widespread micronutrient deficiencies. We in this review have estimated the effect of Micronutrient Powders (MNPs) on the health outcomes of women and children.
\end{abstract}

Methods: We systematically reviewed literature published up to November 2012 to identify studies describing the effectiveness of MNPs. We used a standardized abstraction and grading format to estimate the effect of MNPs by applying the standard Child Health Epidemiology Reference Group (CHERG) rules.

Results: We included 17 studies in this review. MNPs significantly reduced the prevalence of anemia by 34\% (RR: 0.66, 95\% Cl: $0.57-0.77$ ), iron deficiency anemia by 57\% (RR: 0.43 , 95\% Cl: $0.35-0.52$ ) and retinol deficiency by $21 \%$ (RR: $0.79,95 \%$ Cl: $0.64,0.98)$. It also significantly improved the hemoglobin levels (SMD: 0.98, 95\% Cl: 0.55-1.40). While there were no statistically significant impacts observed for serum ferritin and zinc deficiency. Our analysis shows no impact of MNPs on various anthropometric outcomes including stunting (RR: $0.92,95 \%$ Cl: $0.81,1.04$ ), wasting (RR: 1.13, 95\% Cl: 0.91, 1.40), underweight (RR:0.96, 95\% Cl: 0.83, 1.10), HAZ (SMD: 0.04, 95\% Cl: -0.13, 0.22), WAZ (SMD: $0.05,95 \%$ Cl: $-0.12,0.23$ ) and WHZ (SMD: $0.04,95 \%$ Cl: $-0.13,0.21$ ), although showing favorable trends. MNPs were found to be associated with significant increase in diarrhea (RR: 1.04, 95\% Cl: 1.01, 1.06) with nonsignificant impacts on fever and URI.

Conclusion: Our analysis of the effect of MNPs in children suggests benefit in improving anemia and hemoglobin however the lack of impact on growth and evidence of increased diarrhea requires careful consideration before recommending the intervention for implementing at scale.

\section{Introduction}

More than 3.5 million women and children under five die each year in poor countries due to underlying undernutrition [1]. An estimated 178 million children under five are stunted and 55 million children are wasted [2]. Of these stunted children, 160 million (90\%) live in just 36 countries, representing almost half of the children in those countries [2] and many of these children have concomitant micronutrient deficiencies. Deficiencies in vitamin $\mathrm{A}$, iron, zinc and iodine are the most prevalent, accounting for $11 \%$ of global disease burden [3]. The World Health Organization (WHO) estimates

\footnotetext{
* Correspondence: zulfiqar.bhutta@aku.edu

'Division of Women \& Child Health, The Aga Khan University, Karachi, Pakistan

Full list of author information is available at the end of the article
}

that of the roughly two billion people suffering from micronutrient deficiencies, $85 \%$ live in resource poor settings [4] and these often occur as multiple rather than single micronutrient deficiencies [5]. The prevalence is especially high in Southeast Asia and sub-Saharan Africa.

Iron deficiency is widespread and globally about 1.62 billion people are anemic with the highest prevalence among preschool children (47\%) followed by pregnant women (42\%) [6]. Iodine deficiency (IDD) is a public health problem in 130 countries and affects $13 \%$ of world's population [7]. Globally about 740 million people are affected by goiter, and over two billions are considered at risk of IDD. It is estimated that one-third of the world population live in countries with a high prevalence of zinc deficiency. Clinical Vitamin A Deficiency
C Biomed Central

() 2013 Salam et al; licensee BioMed Central Ltd. This is an Open Access article distributed under the terms of the Creative Commons Attribution License (http://creativecommons.org/licenses/by/2.0), which permits unrestricted use, distribution, and reproduction in any medium, provided the original work is properly cited. 
(VAD) affects at least 2.80 million preschool children in over 60 countries, and sub clinical VAD is considered a problem for at least 251 million that includes school-age children and pregnant women [8].

Micronutrients play a critical role in cellular and humoral immune responses, cellular signaling and function, learning and cognitive functions, work capacity, reproductive health and even in the evolution of microbial virulence $[9,10]$. Infants, children and pregnant women have high demands for vitamins and minerals because of increased growth and metabolic requirements and yet their dietary intake often fails to meet these requirements $[3,11]$. In children these micronutrient deficiencies can cause anemia [12], restrict growth [13] and hamper motor and cognitive development [14] and also effect the immune function [15]. Under nutrition in children and women leaves a long term impact on population health and productivity.

Several strategies have been employed to supplement micronutrients to women and children [16-19]. These include nutrition education, dietary modification, food provision, supplementation and fortification. In the last decade point of use or home fortification of maternal and child diets has emerged to tackle the widespread micronutrient deficiencies. Multiple Micronutrient Powders (MNPs) or Sprinkles are powdered encapsulated vitamins and minerals that can be added to prepared foods with little change to the food's taste or texture. MNPs are designed to provide the recommended daily nutrient intake of 2 or more vitamins and minerals to their target populations.

Despite the wide body of primary research on MNP interventions, there are few syntheses of the existing data. A recent Cochrane review has established that MNPs appear effective for reducing anemia and iron deficiency for children under 2 years of age [20]. We in this review have estimated the effect of these MNPs on the health of women and children. We have reviewed the available literature and evaluated the quality of included studies according to the Child Health Epidemiology Group (CHERG) adaptation of Grading of Recommendations, Assessments, Development and Education (GRADE) criteria [21].

\section{Methods}

We systematically reviewed literature published up to November 2012 to identify studies describing the effectiveness of MNPs. Following CHERG Systematic Review Guidelines [21], we searched PubMed, Cochrane Libraries, Embase, and WHO Regional Databases to identify all published and unpublished trials. Additional studies were identified by hand searching references from included studies. Search terms included combinations of Micronutrient* OR "multiple micronutrient" OR "multi-vitamin" OR "multi-mineral" OR "micronutrient powder" OR MNP OR sprinkle AND Fortifi* OR "food fortifi" OR "point of use" OR "home fortification". No language or date restrictions were applied in the searches.

\section{Inclusion criteria}

MNPs were identified as point-of-use powders with two or more micronutrients in their formulation. Studies were included that provided MNPs either in the home or at designated centers, using different multiple micronutrient formulations, with different dosages and duration. Studies that included supporting interventions such as nutrition education were included only if the supporting interventions were given to both the intervention and comparison groups, so that the difference between the two groups was solely of MNPs. Because of the unique nature of this intervention and a need to do a separate analysis specifically for this intervention, we excluded studies examining the impact of supplementary food provision, lipid-based supplements, micronutrient crushable tablets or foodlets, fortified milk or complementary foods and other fortified foods and beverages including fortified seasoning powders.

\section{Abstraction, analysis and summary measure}

We abstracted data describing study identifiers and context, study design and limitations, intervention specifics and outcome effects into a standardized abstraction form for studies that met the final inclusion criteria as detailed in the CHERG Systematic Review Guidelines [21]. Outcomes of interest included hematological; anemia, hemoglobin levels, serum micronutrient levels, anthropometric; stunting, wasting, underweight, weight for age z-score (WAZ), height for age z- score (HAZ), weight for height $\mathrm{z}$-score (WHZ), head circumference and morbidity; diarrhea, upper respiratory infections (URI), fever and mortality among women and children. Each study was assessed and graded according to the CHERG adaptation of the GRADE technique [21].

\section{Quantitative data synthesis}

We conducted a meta-analysis for individual studies and pooled statistics were reported as the relative risk (RR) for categorical variables and standard mean difference (SMD) for continuous variables between the experimental and control groups with 95\% confidence intervals (CI). Mantel-Haenszel pooled RR and corresponding 95\% CI were reported or the DerSimonian-Laird pooled $\mathrm{RR}$ and corresponding 95\% CI where there was an unexplained heterogeneity. All analyses were conducted using the software Review Manager 5.1. Heterogeneity was quantified by $\mathrm{Chi}^{2}$ and $\mathrm{I}^{2}$, which can be interpreted as the percentage of the total variation between studies 
that is attributable to heterogeneity rather than to chance, a low p-value (less than 0.1 ) or a large chisquared statistic relative to its degree of freedom and $\mathrm{I}^{2}$ values greater than $50 \%$ were taken as substantial and high heterogeneity. In situations of high heterogeneity, causes were explored by sensitivity analysis and random effect models were used.

We summarized the evidence by outcome, including qualitative assessments of study quality and quantitative measures, according to the standard guidelines. A grade of "high", "moderate", "low" and "very low" was used for grading the overall evidence indicating the strength of an effect on specific health outcome according to the CHERG Rules for Evidence Review [21].

\section{Results}

We identified 2556 titles from search conducted in all databases. After screening titles and abstracts, we reviewed 26 papers for the identified outcome measures of interest of which 11 papers investigated either multiple micronutrient spreads or seasonings and were excluded from this review and 17 [22-38] studies were finally selected for inclusion which evaluated the impact of MNP versus no intervention or control and reported the outcomes of interest (Figure 1). Most of the studies were done on children aged 6 months to 6 years of age, while two studies had children up to 11 years of age. All studies were conducted in developing countries. There were no studies identified which were on women and met our inclusion criteria. None of the included studies reported on the outcome of mortality. Table 1 shows the characteristics of the included studies.
In Table 2 and 3, we report the quality assessment of studies by outcomes. All the evidence was of moderate outcome specific quality. For the hematologic indicators (Table 2), the findings were based on 15 studies. MNPs significantly reduced the prevalence of anemia by $34 \%$ (RR: 0.66, 95\% CI: 0.57-0.77) (Figure 2), iron deficiency anemia by $57 \%$ (RR: $0.43,95 \%$ CI: $0.35-0.52$ ) and retinol deficiency by $21 \%$ (RR: $0.79,95 \%$ CI: $0.64,0.98$ ). It also significantly improved the hemoglobin levels (SMD: 0.98, 95\% CI: 0.55-1.40) (Figure 3). MNPs did not show a significant improvement in serum ferritin concentration and zinc deficiency.

For the anthropometric outcomes (Table 3), data was pooled for six studies. MNPs did not show a significant improvement in any of the anthropometric outcomes including stunting (RR: 0.92, 95\% CI: 0.81, 1.04), wasting (RR: 1.13, 95\% CI: 0.91, 1.40), underweight (RR:0.96, 95\% CI: 0.83, 1.10), HAZ (SMD: 0.04, 95\% CI: -0.13, 0.22), WAZ (SMD: $0.05,95 \%$ CI: $-0.12,0.23$ ) and WHZ (SMD: 0.04, 95\% CI: -0.13, 0.21), although showing favorable trends, as the direction of effect was on the positive side although non-significant.

For the morbidity outcomes (Table 3), data from four studies was pooled. MNPs were associated with significant increase in the incidence of diarrhea (RR: 1.04, 95\% CI: 1.01, 1.06) (Figure 4), while there was no significant rise in recurrent diarrhea (RR: 2.86, 95\% CI: 0.12-69.0), fever (RR: 1.03, 95\% CI: 0.70, 1.51) and URI (RR: 1.17, 95\% CI: 0.71, 1.92).

\section{Recommendation for the LiST model}

Of the outcomes assessed for the effect of MNPs in children, we applied the CHERG rules for evidence review

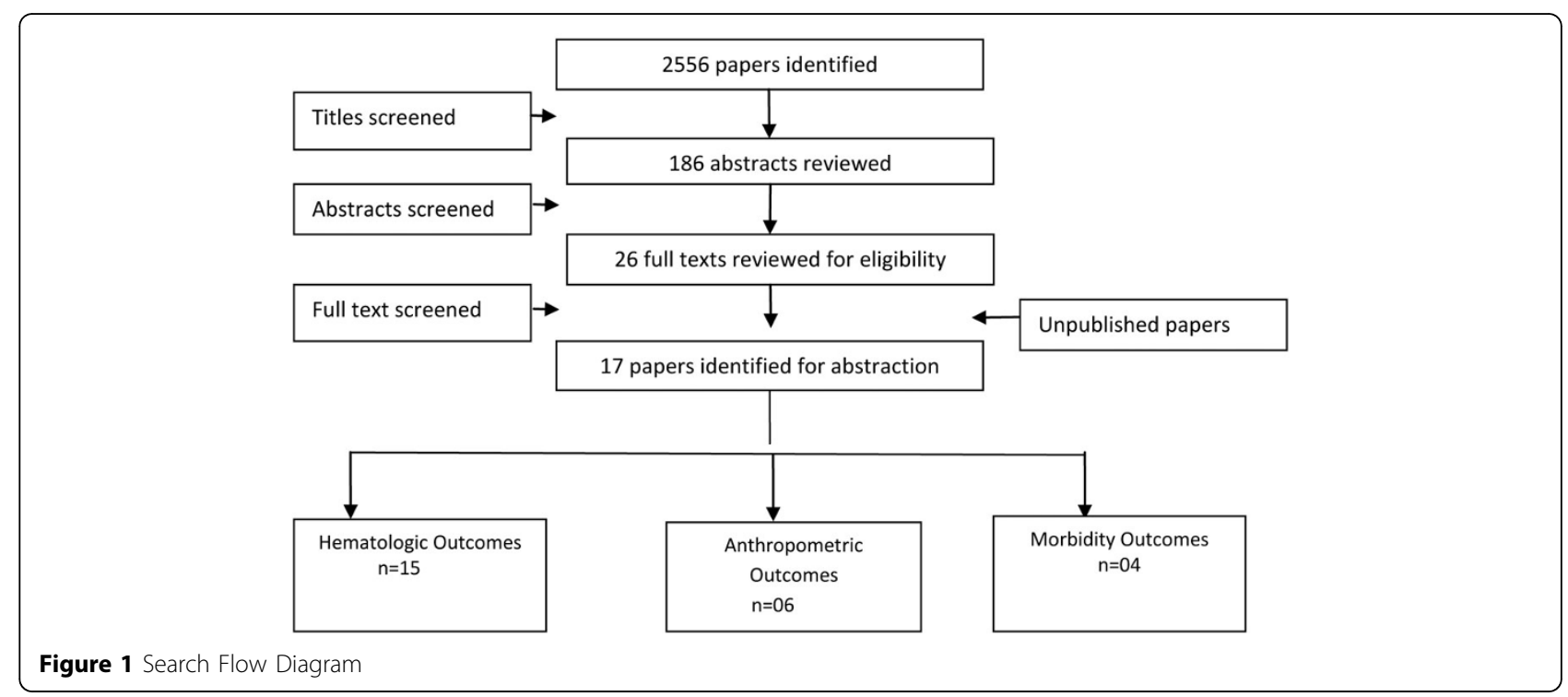


Table 1 Characteristics of included studies

\begin{tabular}{|c|c|c|c|c|}
\hline Study & Country & Target Group & MNP Composition & Duration \\
\hline Adu-Afarwuah 2007 & Ghana & 6-12 month olds & $\begin{array}{c}\text { B-Carotene-300 } \mu \mathrm{g} \text { RE, Vitamin C-50 mg, Vitamin D3-7.5 } \mu \mathrm{g} \text {, Folic acid- } \\
150 \mu \mathrm{g} \text {, Iron (Fumarate)- } 12.5 \mathrm{mg} \text {, Zinc (Gluconate)- } 5 \mathrm{mg}\end{array}$ & 1 year \\
\hline Adu-Afarwuah 2008 & Ghana & 6-12 month olds & $\begin{array}{l}\text { B-Carotene-300 } \mu \mathrm{g} \text { RE, Vitamin C-50 mg, Vitamin D3- } 7.5 \mu \mathrm{g} \text {, Folic acid- } \\
150 \mu \mathrm{g} \text {, Iron (Fumarate)-12.5 mg, Zinc (Gluconate)- } 5 \mathrm{mg}\end{array}$ & 1 year \\
\hline Agostoni 2007 & Cambodia & 6 month olds & $\begin{array}{l}\text { Fe- } 12.5 \mathrm{mg} \text { (fumarate), Zn- } 5 \mathrm{mg} \text { (gluconate), Vitamin C - 50mg, Vitamin A - } \\
\text { 300mg, Vitamin D3-7.5mg, Folic acid - } 150 \mathrm{mg} \text {, Potato maltodextrins SQ to } 1 \mathrm{~g} \text {. }\end{array}$ & 1 year \\
\hline Kounnavong 2011 & $\begin{array}{l}\text { Lao People's } \\
\text { Democratic } \\
\text { Republic }\end{array}$ & 6-52 month olds & 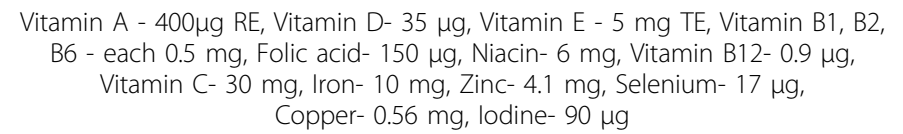 & 6 months \\
\hline Kumar 2007 & India & 7-11 year olds & $\begin{array}{c}\text { Vitamin A } 1500 \mathrm{lU} / \mathrm{g} \text {, Vitamin B2, B6, B12 each -1 mg/g, Calcium pentothenate- } \\
1 \mathrm{mg} / \mathrm{g} \text {, Niacin - } 15 \mathrm{mg} / \mathrm{g} \text {, Folic acid-100 mcg/g, Vitamin E -30 IU/g, Vitamin C- } \\
30 \mathrm{mg} / \mathrm{g} \text {, Iron - } 10 \mathrm{mg} / \mathrm{g} \text {, Lysine - } 250 \mathrm{mg} / \mathrm{g} \text {, Calcium - } 15.63 \%\end{array}$ & 1 year \\
\hline Lundeen 2010 & Kyrgyzistan & 6-36 months old & $\begin{array}{l}\text { Elemental iron (fumarate)- } 12.5 \mathrm{mg} \text {, Vitamin A - } 300 \mu \mathrm{g} \text {, Zinc (gluconate)- } \\
5 \mathrm{mg} \text {, Vitamin C (ascorbic acid) - } 30 \text { mg, Folic acid- } 160 \mu \mathrm{g}\end{array}$ & 2 months \\
\hline Macharia-Mutie 2012 & Kenya & 1-5 year olds & 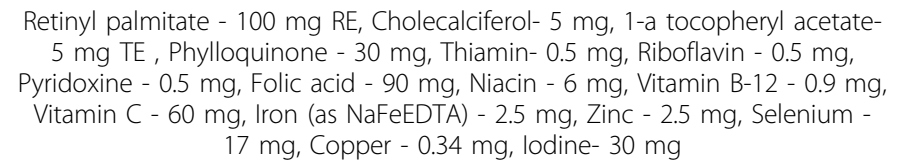 & 4 months \\
\hline Menon 2007 & Haiti & 9-24 month olds & $\begin{array}{c}\text { Iron- } 12.5 \text { mg, Zinc- } 5 \mathrm{mg}, \text { Vitamin A- } 400 \mathrm{mg} \text {, Folic acid - 160mg, } \\
\text { Vitamin C- } 30 \mathrm{mg}\end{array}$ & 2 months \\
\hline Osei 2010 & India & 6-10 year olds & $\begin{array}{l}\text { Iron (NaFeEDTA)- } 10 \mathrm{mg} \text {, Vitamin A (retinyl acetate)- } 375 \mathrm{mg} \text {, Zinc (zinc } \\
\text { gluconate) -4.2 mg, Folic acid - } 225 \mathrm{mg} \text {, lodine (potassium iodide) - } 90 \mathrm{mg} \text {, } \\
\text { Vitamin C (ascorbic acid) - } 26.25 \mathrm{mg} \text {, Thiamine (thiamine mononitrate)- } 0.68 \\
\text { mg, Riboflavin- } 0.68 \mathrm{mg} \text {, Niacin (nicotinamide) - } 9 \mathrm{mg} \text {, Vitamin B-12- } 1.35 \mathrm{mg} \text {, } \\
\text { Vitamin B-6- } 0.75 \mathrm{mg} \text {, Vitamin D (ergocalciferol) - } 3.75 \mathrm{mg} \text {, } \\
\text { Vitamin E - } 5.25 \mathrm{mg} \text {, Copper [CuSO4.(H2O)5]- } 0.45 \mathrm{mg}\end{array}$ & 8 months \\
\hline Sharieff 2006 & Pakistan & $6-12$ month olds & $\begin{array}{c}\text { Zinc gluconate- } 5 \mathrm{mg} \text {, Ferrous fumarate - } 30 \mathrm{mg} \text {, Vitamin C - } 50 \mathrm{mg} \text {, Vitamin } \\
\text { A - } 300 \mathrm{mg} \text {, Vitamin D3 - } 7.5 \mathrm{mg} \text {, Folic acid- } 150 \mathrm{mg}\end{array}$ & 2 months \\
\hline Varma 2007 & India & $36-66$ month olds & Ferrous fumarate- 14 mg, Vitamin A- $500 \mathrm{IU}$, Folic acid- $0.05 \mathrm{mg}$ & 6 months \\
\hline Giovannini 2006 & Cambodia & 6 month olds & $\begin{array}{c}\text { Fe (iron II fumarate) - } 12.5 \mathrm{mg}, \mathrm{Zn} \text { (gluconate) - } 5 \mathrm{mg} \text {, Vitamin C - } 50 \mathrm{mg} \text {, } \\
\text { Vitamin A - } 300 \mu \mathrm{g} \text {, Vitamin D3 - } 7.5 \mu \mathrm{g} \text {, Folic acid 50- } 150 \mu \mathrm{g} \text {, Potato } \\
\text { maltodextrins - SQ to } 1 \mathrm{~g}\end{array}$ & 1 year \\
\hline Sharieff 2007 & China & $3-6$ years & $\begin{array}{c}\text { Iron (ferrous fumarate)- } 30 \mathrm{mg} \text {, Zinc gluconate- } 5 \mathrm{mg} \text {, Vitamin C- } 50 \mathrm{mg} \text {, } \\
\text { Vitamin A- } 300 \mathrm{mg} \text {, Vitamin D3 - 7.5mg, Folic acid- } 150 \mathrm{mg}\end{array}$ & 3 months \\
\hline Suchdev 2010 & Kenya & 6-35 month olds & $\begin{array}{c}\text { Ferrous fumarate- } 12.5 \mathrm{mg} \text {, Vitamin A- } 375 \mu \mathrm{g} \text {, Zinc- } 5 \mathrm{mg} \text {, Folic acid- } 150 \mu \mathrm{g} \text {, } \\
\text { Vitamin C- } 35 \mathrm{mg} \text {, Vitamin D3 - } 5 \mu \mathrm{g} \text {, Vitamin E- } 6 \mathrm{mg} \text {, Niacin- } 6 \mathrm{mg} \text {, Copper- } \\
0.6 \mathrm{mg} \text {, lodine - } 50 \mu \mathrm{g} \text {, Thiamine, riboflavin and vitamin B-6 - } 0.5 \mathrm{mg} \text {, Vitamin } \\
\text { B-12- } 0.9 \mathrm{mg}\end{array}$ & 1 year \\
\hline Jack 2012 & Cambodia & 6 month olds & 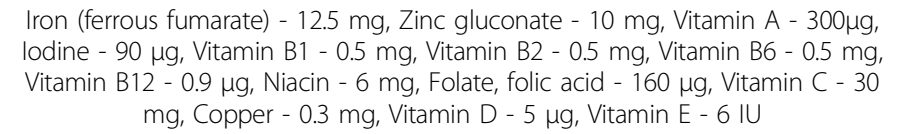 & 18 months \\
\hline Bhutta (unpublished) & Pakistan & 6-18 months & $\begin{array}{l}\text { Ferrous fumarate- } 12.5 \mathrm{mg} \text {, Vitamin C - } 50 \mathrm{mg} \text {, Vitamin A (retinol acetate)- } \\
300 \mu \mathrm{g} \text {, Vitamin D - } 5 \mu \mathrm{g} \text {, Folic acid - } 150 \mu \mathrm{g} \text {, Zinc gluconate- } 10 \mathrm{mg}\end{array}$ & 24 months \\
\hline
\end{tabular}

to these outcomes. There was no data on mortality and the evidence on anthropometric outcomes is weak. With the current available evidence, we suggest that MNPs in children is associated with a $34 \%$ decrease in the incidence of anemia. The evidence of increased diarrhea suggests careful evaluation of the associated risks.

\section{Discussion}

In this systematic review our objective was to summarize the effect of MNPs on the health outcomes of women and children. We did not find any study reporting outcomes on women and seventeen studies were included that reported on various outcomes on children. The studies contributing data in this review were conducted in developing countries hence increasing the generalizability of the studies to children in low and middle income countries with the highest undernutrition rates. Most of the studies were effectiveness trials evaluating the impact of MNPs in community settings. All of the studies were on children 
Table 2 Quality Assessment by Hematologic Outcome

\begin{tabular}{|c|c|c|c|c|c|c|c|c|}
\hline & \multicolumn{5}{|c|}{ Quality Assessment } & \multicolumn{3}{|c|}{ Summary of Findings } \\
\hline & \multicolumn{2}{|r|}{ Directness } & \multicolumn{2}{|c|}{ No of events } & \multirow[b]{2}{*}{$\begin{array}{l}\text { Generalizability to } \\
\text { intervention of interest }\end{array}$} & \multirow[b]{2}{*}{ Intervention } & \multirow[b]{2}{*}{ Control } & \multirow[b]{2}{*}{$\begin{array}{l}\mathrm{RR} / \mathrm{SMD} \\
(95 \% \mathrm{Cl})\end{array}$} \\
\hline No of studies & Design & Limitations & Consistency & $\begin{array}{c}\text { Generalizability to } \\
\text { population of } \\
\text { interest } \\
\end{array}$ & & & & \\
\hline \multicolumn{9}{|c|}{ Anemia: Moderate outcome specific quality of evidence } \\
\hline Eleven & RCT & $\begin{array}{c}\text { Significant } \\
\text { heterogeneity, random } \\
\text { effect model used }\end{array}$ & $\begin{array}{l}\text { Six of ten } \\
\text { studies } \\
\text { suggest } \\
\text { benefit }\end{array}$ & $\begin{array}{l}\text { All studies from } \\
\text { the developing } \\
\text { countries }\end{array}$ & $\begin{array}{l}\text { The duration of the } \\
\text { studies ranged from 2- } \\
12 \text { months and the age } \\
\text { of the children from } 6 \\
\text { months to } 10 \text { years. }\end{array}$ & 1081 & 1443 & $\begin{array}{c}\text { RR: } 0.66 \\
{[0.57,0.77]}\end{array}$ \\
\hline \multicolumn{9}{|c|}{ Iron deficiency Anemia: Moderate outcome specific quality of evidence } \\
\hline $\begin{array}{l}\text { Seven studies } \\
\text { (six data sets) }\end{array}$ & $\mathrm{RCT}$ & $\begin{array}{c}\text { Significant } \\
\text { heterogeneity, random } \\
\text { effect model used }\end{array}$ & $\begin{array}{l}\text { Four of six } \\
\text { studies } \\
\text { suggest } \\
\text { benefit }\end{array}$ & $\begin{array}{l}\text { All studies from } \\
\text { the developing } \\
\text { countries }\end{array}$ & $\begin{array}{l}\text { The duration of the } \\
\text { studies ranged from } \\
2-12 \text { months }\end{array}$ & 404 & 986 & $\begin{array}{c}\text { RR: } 0.43 \\
{[0.35,0.52]}\end{array}$ \\
\hline \multicolumn{9}{|c|}{ Hemoglobin: Moderate outcome specific quality of evidence } \\
\hline $\begin{array}{l}\text { Fourteen } \\
\text { studies } \\
\text { (Fifteen data } \\
\text { sets) }\end{array}$ & $\mathrm{RCT}$ & $\begin{array}{c}\text { Significant } \\
\text { heterogeneity, random } \\
\text { effect model used }\end{array}$ & $\begin{array}{l}\text { Nine studies } \\
\text { suggest } \\
\text { benefit }\end{array}$ & $\begin{array}{l}\text { All studies from } \\
\text { the developing } \\
\text { countries }\end{array}$ & $\begin{array}{c}\text { Studies ranged in } \\
\text { duration from 2-12 } \\
\text { months. }\end{array}$ & 4571 & 3783 & $\begin{array}{l}\text { SMD: } 0.98 \\
{[0.55,0.40]}\end{array}$ \\
\hline \multicolumn{9}{|c|}{ Serum Zinc: Moderate outcome specific quality of evidence } \\
\hline Three & RCT & $\begin{array}{c}\text { Significant } \\
\text { heterogeneity so a } \\
\text { random effect model } \\
\text { used }\end{array}$ & $\begin{array}{l}\text { One study } \\
\text { suggested } \\
\text { benefit }\end{array}$ & $\begin{array}{l}\text { All studies from } \\
\text { developing } \\
\text { countries }\end{array}$ & $\begin{array}{l}\text { One study was } \\
\text { conducted in school }\end{array}$ & 761 & 788 & $\begin{array}{l}\text { SMD: }-0.22 \\
{[-0.52,0.09]}\end{array}$ \\
\hline \multicolumn{9}{|c|}{ Serum Retinol: Moderate outcome specific quality of evidence } \\
\hline Two & $\mathrm{RCT}$ & $\begin{array}{c}\text { Significant } \\
\text { heterogeneity so a } \\
\text { random effect model } \\
\text { used }\end{array}$ & $\begin{array}{l}\text { One study } \\
\text { suggest } \\
\text { benefit }\end{array}$ & $\begin{array}{l}\text { Both studies from } \\
\text { India }\end{array}$ & $\begin{array}{l}\text { Study duration ranged } \\
\text { from 6-8 months. }\end{array}$ & 464 & 504 & $\begin{array}{l}\text { SMD: } 1.66 \\
{[-1.60,4.92]}\end{array}$ \\
\hline \multicolumn{9}{|c|}{ Serum Ferritin: Moderate outcome specific quality of evidence } \\
\hline Four & RCT & $\begin{array}{c}\text { Significant } \\
\text { heterogeneity, random } \\
\text { effect model used }\end{array}$ & $\begin{array}{c}\text { Three studies } \\
\text { suggest } \\
\text { benefit }\end{array}$ & $\begin{array}{l}\text { All studies from } \\
\text { developing } \\
\text { countries }\end{array}$ & $\begin{array}{c}\text { Studies ranged in } \\
\text { duration from } 6 \text { months } \\
\text { to } 12 \text { months }\end{array}$ & 850 & 884 & $\begin{array}{l}\text { SMD: } 1.78 \\
{[-0.31,3.88]}\end{array}$ \\
\hline \multicolumn{9}{|c|}{ Zinc Deficiency: Moderate outcome specific quality of evidence } \\
\hline Two & RCT & $\begin{array}{l}\text { No significant } \\
\text { heterogeneity, fixed } \\
\text { effect model used }\end{array}$ & $\begin{array}{l}\text { None of the } \\
\text { study suggests } \\
\text { benefit }\end{array}$ & $\begin{array}{l}\text { All studies from } \\
\text { the developing } \\
\text { countries }\end{array}$ & $\begin{array}{l}\text { The study duration } \\
\text { ranged from 6-8 } \\
\text { months. }\end{array}$ & 258 & 272 & $\begin{array}{l}\text { RR: } 1.02 \\
{[0.87,1.19]}\end{array}$ \\
\hline \multicolumn{9}{|c|}{ Retinol Deficiency: Moderate outcome specific quality of evidence } \\
\hline Three & RCT & $\begin{array}{l}\text { No significant } \\
\text { heterogeneity, fixed } \\
\text { effect model used }\end{array}$ & $\begin{array}{l}\text { None of the } \\
\text { study suggests } \\
\text { benefit }\end{array}$ & $\begin{array}{l}\text { All studies from } \\
\text { the developing } \\
\text { countries }\end{array}$ & $\begin{array}{l}\text { The study duration } \\
\text { ranged from 6-12 } \\
\text { months. }\end{array}$ & 111 & 145 & $\begin{array}{c}\text { RR: } 0.79 \\
{[0.64,0.98]}\end{array}$ \\
\hline
\end{tabular}

less than six years of age, except two studies [30,39] that included children over 6 years of age although the subgroup analysis for children under five did not show any difference in the findings. Clinical heterogeneity was observed due to variations in type of intervention (number of micronutrients used ranged from 3 to 15), duration of the intervention (2-24 months), target population and different time intervals for follow-up. All the MNPs used contained iron in their composition.
The intervention was mostly reported to be acceptable by the mothers and children and there was no major loss to follow-up reported due to the intervention in any of the included studies. There have been no adverse events identified by any study except one [38] that reported increased diarrhea in the intervention group compared to control.

This review shows that MNPs raise serum hemoglobin levels and reduce anemia significantly, but the evidence on growth is weak, as relatively few studies have evaluated 
Table 3 Quality assessment by anthropometric and morbidity outcomes

\begin{tabular}{|c|c|c|c|c|c|c|c|c|}
\hline \multicolumn{9}{|c|}{ Stunting: Moderate outcome specific quality of evidence } \\
\hline $\begin{array}{l}\text { Two study } \\
\text { (three data } \\
\text { sets) }\end{array}$ & $\mathrm{RCT}$ & $\begin{array}{l}\text { No significant } \\
\text { heterogeneity, fixed } \\
\text { effect model used }\end{array}$ & $\begin{array}{l}\text { None of the study } \\
\text { suggests benefit }\end{array}$ & $\begin{array}{l}\text { All studies from } \\
\text { the developing } \\
\text { countries }\end{array}$ & & 810 & 838 & $\begin{array}{l}\text { RR: } 0.92 \\
{[0.81,1.04]}\end{array}$ \\
\hline \multicolumn{9}{|c|}{ Wasting: Moderate outcome specific quality of evidence } \\
\hline $\begin{array}{l}\text { Two study } \\
\text { (three data } \\
\text { sets) }\end{array}$ & $\mathrm{RCT}$ & $\begin{array}{l}\text { No significant } \\
\text { heterogeneity, fixed } \\
\text { effect model used }\end{array}$ & $\begin{array}{l}\text { None of the study } \\
\text { suggests benefit }\end{array}$ & $\begin{array}{l}\text { All studies from } \\
\text { the developing } \\
\text { countries }\end{array}$ & & 263 & 234 & $\begin{array}{l}\text { RR: } 1.13 \\
{[0.91,1.40]}\end{array}$ \\
\hline \multicolumn{9}{|c|}{ Underweight: Moderate outcome specific quality of evidence } \\
\hline $\begin{array}{l}\text { Three } \\
\text { studies (four } \\
\text { data sets) }\end{array}$ & $\mathrm{RCT}$ & $\begin{array}{l}\text { No significant } \\
\text { heterogeneity, fixed } \\
\text { effect model used }\end{array}$ & $\begin{array}{l}\text { None of the studies } \\
\text { suggest significant } \\
\text { benefit }\end{array}$ & $\begin{array}{l}\text { All the studies } \\
\text { from developing } \\
\text { countries }\end{array}$ & $\begin{array}{l}\text { All studies included more than } 3 \\
\text { micronutrients. The studies ranged } \\
\text { in duration from } 4-12 \text { months. }\end{array}$ & 671 & 679 & $\begin{array}{l}\text { RR: } 0.96 \\
{[0.83,1.10]}\end{array}$ \\
\hline \multicolumn{9}{|c|}{ HAZ: Moderate outcome specific quality of evidence } \\
\hline Three & $\mathrm{RCT}$ & $\begin{array}{l}\text { No significant } \\
\text { heterogeneity, fixed } \\
\text { effect model used }\end{array}$ & $\begin{array}{l}\text { None of the studies } \\
\text { suggest significant } \\
\text { benefit }\end{array}$ & $\begin{array}{l}\text { All studies are } \\
\text { from Africa }\end{array}$ & $\begin{array}{l}\text { All studies included more than } 3 \\
\text { micronutrients. The studies ranged } \\
\text { in duration from 6-12 months. }\end{array}$ & 271 & 253 & $\begin{array}{l}\text { SMD: } 0.04 \\
{[-0.13,0.22]}\end{array}$ \\
\hline \multicolumn{9}{|c|}{ WAZ: Moderate outcome specific quality of evidence } \\
\hline Three & $\mathrm{RCT}$ & $\begin{array}{l}\text { No significant } \\
\text { heterogeneity, fixed } \\
\text { effect model used }\end{array}$ & $\begin{array}{l}\text { None of the studies } \\
\text { suggest significant } \\
\text { benefit }\end{array}$ & $\begin{array}{l}\text { All studies from } \\
\text { Africa }\end{array}$ & $\begin{array}{l}\text { All studies included more than } 3 \\
\text { micronutrients. The studies ranged } \\
\text { in duration from 6-12 months. }\end{array}$ & 271 & 253 & $\begin{array}{l}\text { SMD: } 0.05 \\
{[-0.12,0.23]}\end{array}$ \\
\hline \multicolumn{9}{|c|}{ WHZ: Moderate outcome specific quality of evidence } \\
\hline Three & $\mathrm{RCT}$ & $\begin{array}{l}\text { No significant } \\
\text { heterogeneity, fixed } \\
\text { effect model used }\end{array}$ & $\begin{array}{l}\text { None of the studies } \\
\text { suggest significant } \\
\text { benefit }\end{array}$ & $\begin{array}{l}\text { All studies from } \\
\text { Africa }\end{array}$ & $\begin{array}{l}\text { All studies included more than } 3 \\
\text { micronutrients. The studies ranged } \\
\text { in duration from } 6-12 \text { months. }\end{array}$ & 271 & 253 & $\begin{array}{l}\text { SMD: } 0.04 \\
{[-0.13,0.21]}\end{array}$ \\
\hline \multicolumn{9}{|c|}{ Diarrhea: Moderate outcome specific quality of evidence } \\
\hline $\begin{array}{l}\text { Four studies } \\
\text { (five data } \\
\text { sets) }\end{array}$ & RCT & $\begin{array}{l}\text { No significant } \\
\text { heterogeneity, fixed } \\
\text { effect model used }\end{array}$ & $\begin{array}{l}\text { Direction of } \\
\text { evidence is } \\
\text { consistent across } \\
\text { studies }\end{array}$ & $\begin{array}{l}\text { All the studies } \\
\text { from developing } \\
\text { countries }\end{array}$ & $\begin{array}{l}\text { Number of micronutrients ranged } \\
\text { from 5-15. Duration varied from } 2-12 \\
\text { months. One of the studies targeted } \\
\text { children in school. }\end{array}$ & 1692 & 1679 & $\begin{array}{l}\text { RR: } 1.04 \\
{[1.01,1.06]}\end{array}$ \\
\hline \multicolumn{9}{|c|}{ Recurrent Diarrhea: Moderate outcome specific quality of evidence } \\
\hline One & $\mathrm{RCT}$ & Only one study & & $\begin{array}{l}\text { Study was } \\
\text { conducted in } \\
\text { Cambodia }\end{array}$ & $\begin{array}{l}\text { Study was conducted over a } 12 \\
\text { month period }\end{array}$ & 1 & 0 & $\begin{array}{l}\text { RR: } 2.86 \\
{[0.12} \\
69.00]\end{array}$ \\
\hline \multicolumn{9}{|c|}{ URI: Moderate outcome specific quality of evidence } \\
\hline Two & $\mathrm{RCT}$ & $\begin{array}{l}\text { No significant } \\
\text { heterogeneity, fixed } \\
\text { effect model used }\end{array}$ & $\begin{array}{l}\text { None of the studies } \\
\text { suggest benefit }\end{array}$ & $\begin{array}{l}\text { All the studies } \\
\text { from developing } \\
\text { countries }\end{array}$ & $\begin{array}{l}\text { Number of micronutrients ranged } \\
\text { from 5-14. Duration varied from 8-12 } \\
\text { months. One of the studies targeted } \\
\text { children in school. }\end{array}$ & 30 & 26 & $\begin{array}{l}\text { RR: } 1.17 \\
{[0.71,1.92]}\end{array}$ \\
\hline \multicolumn{9}{|c|}{ Fever: Moderate outcome specific quality of evidence } \\
\hline One & $\mathrm{RCT}$ & Only one study & & $\begin{array}{l}\text { Study was } \\
\text { conducted in } \\
\text { India }\end{array}$ & & 41 & 41 & $\begin{array}{l}\text { RR: } 1.03 \\
{[0.70,1.51]}\end{array}$ \\
\hline
\end{tabular}

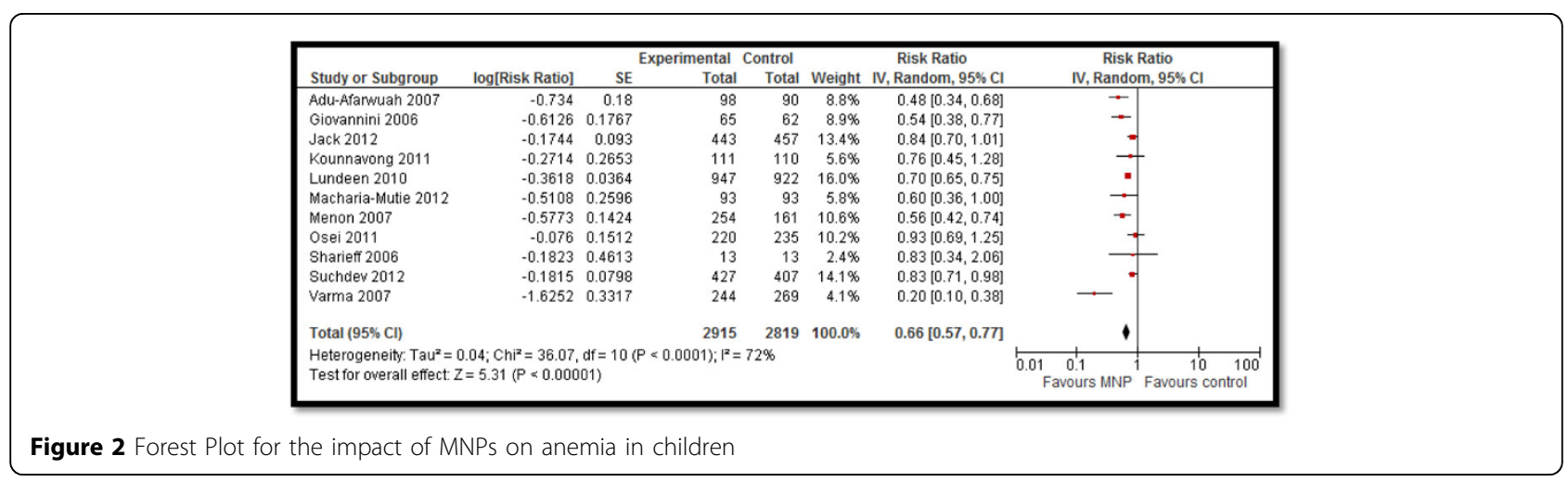




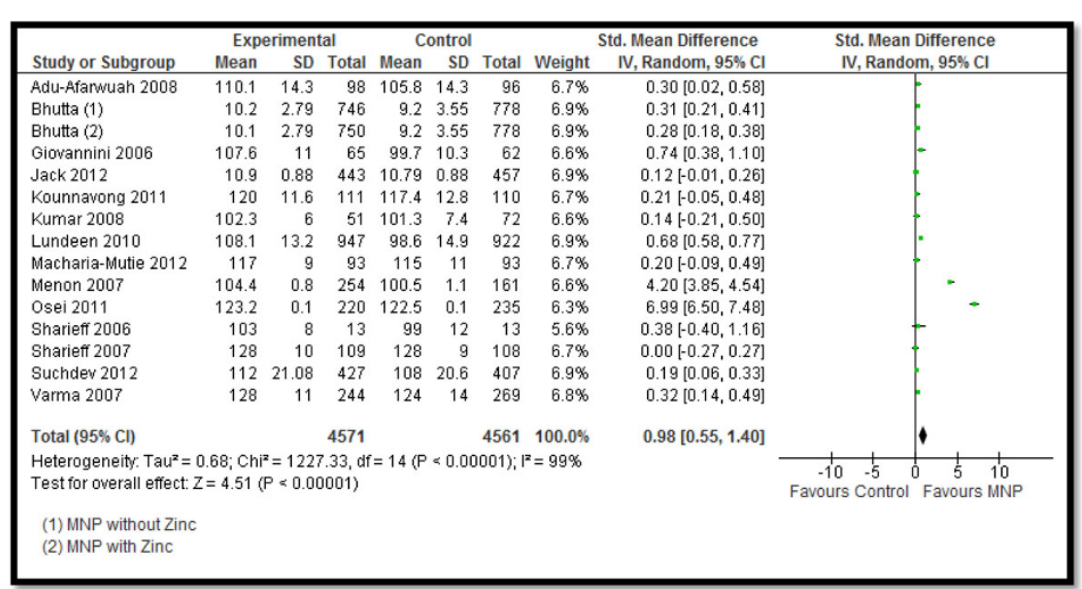

Figure 3 Forest Plot for the impact of MNPs on hemoglobin in children

this outcome. Improved hemoglobin and anemia status could be attributable to the iron component in all the MNPs used. Some studies have reported benefits on other developmental outcomes like walking by 12 months but not on growth [22]. This could be due to relatively shorter duration of the intervention to show actual long term impacts. These findings also suggest that multiple micronutrient interventions alone might not improve growth outcomes. To ensure long term impacts and sustainability, health education that aims to modify food habits would be necessary to improve child growth rates. Also, if the intervention initiation coincides with the child's diet transition from breast feeding to complementary feeding, the results may show improved growth.

The finding of significantly increased diarrhea is potentially alarming. It is mainly based on the significant increase in diarrhea observed in one large trial [38]. The association between increased diarrhea with iron supplementation is well recognized in the literature and is also reported in a review on iron supplementation by Gera [40]. However, our finding of excess morbidity and negligible growth benefit cannot be ignored in settings where large scale use of MNPs is being considered. The increased diarrhea burden could be one of the potential explanations for reduced growth benefits of MNPs.

The evidence is weak for any effect of MNPs on growth, as there were very few studies pooled for each outcome. More research is needed and studies need to report the outcomes of stunting, wasting, morbidity and mortality consistently to strengthen the evidence and evaluate its actual impact on growth and morbidity. A major research gap identified was that there were no studies evaluating the impact on women as all the studies targeted children only.

\section{Conclusion}

Our analysis of the effect of MNPs in children suggests benefit in improving anemia and hemoglobin however there is lack of impact on growth. Evidence of increased diarrhea requires careful consideration before recommending the intervention for implementation at scale.

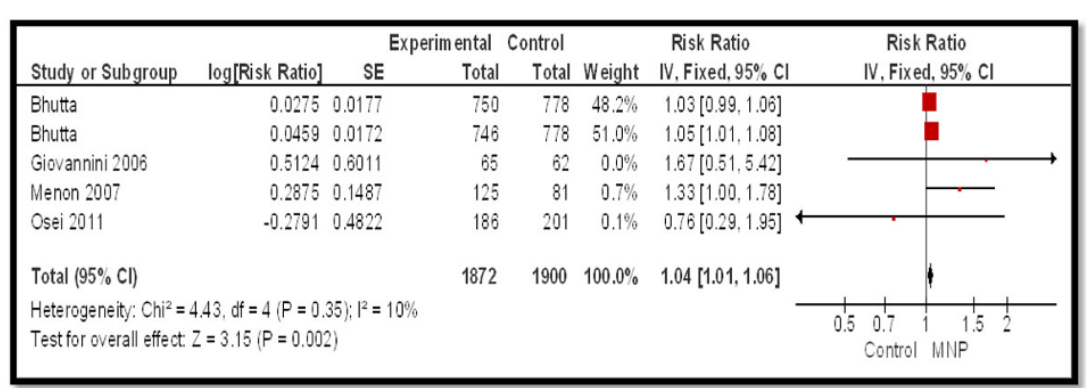

Figure 4 Forest Plot for the impact of MNPs on diarrhea in children 


\section{Competing interests}

We do not have any financial or non-financial competing interests for this review.

\section{Authors' contributions}

Dr. ZAB was responsible for designing the review and coordinating the review. RAS, CM and JKD were responsible for: data collection, screening the search results, screening retrieved papers against inclusion criteria, appraising quality of papers, abstracting data from papers, entering data into RevMan, analysis and interpretation of data and writing the review. ZAB and RAS critically reviewed and modified the manuscript.

\section{Acknowledgment}

This work was supported in part by a grant from the Bill \& Melinda Gates Foundation.

\section{Declarations}

The publication costs for this supplement were funded by a grant from the Bill \& Melinda Gates Foundation to the US Fund for UNICEF (grant 43386 to "Promote evidence-based decision making in designing maternal, neonatal, and child health interventions in low- and middle-income countries"). The Supplement Editor is the principal investigator and lead in the development of the Lives Saved Tool (LiST), supported by grant 43386. He declares that he has no competing interests.

This article has been published as part of BMC Public Health Volume 13 Supplement 3, 2013: The Lives Saved Tool in 2013: new capabilities and applications. The full contents of the supplement are available online at http://www.biomedcentral.com/bmcpublichealth/supplements/13/S3.

\section{Authors' details}

'Division of Women \& Child Health, The Aga Khan University, Karachi, Pakistan. ${ }^{2}$ University of Toronto, Toronto, Ontario, Canada. ${ }^{3}$ Global Child Health and Policy, Centre for Global Child Health, The Hospital for Sick Children, Toronto, ON, Canada.

Published: 17 September 2013

\section{References}

1. WHO: Severe malnutrition: report of a consultation to review current literature. Geneva: World Health Organization; 2000.

2. Bhutta ZA: Micronutrient needs of malnourished children. Curr Opin Clin Nutr Metab Care 2008, 11(3):309-314.

3. Black RE, Allen LH, Bhutta ZA, Caulfield LE, De Onis M, Ezzati M, Mathers $C$, Rivera J: Maternal and child undernutrition: global and regional exposures and health consequences. Lancet 2008, 371(9608):243-260.

4. WHO: World health report. Geneva: World Health Organization; 2000.

5. Abu-Saad K, Fraser D: Maternal nutrition and birth outcomes. Epidemiologic Reviews 2010, 32(1):5-25.

6. Benoist B, McLean E, Egll I, Cogswell M: Worldwide prevalence of anaemia 1993-2005: WHO global database on anaemia. Worldwide prevalence of anaemia 1993-2005: WHO global database on anaemia 2008.

7. Vir SC: Current status of iodine deficiency disorders (IDD) and strategy for its control in India. Indian journal of pediatrics 2002, 69(7):589-596.

8. Stephenson LS, Latham MC, Ottesen EA: Global malnutrition. Parasitology 2000, 121 Suppl:S5-S22.

9. Guerrant RL, Lima AAM, Davidson F: Micronutrients and infection: interactions and implications with enteric and other infections and future priorities. Journal of Infectious Diseases 2000, 182(Supplement 1): S134-S138.

10. Kapil U, Bhavna A: Adverse effects of poor micronutrient status during childhood and adolescence. Nutr Rev 2002, 60(5 Pt 2):S84-S90.

11. Black RE: Micronutrients in pregnancy. British Journal of Nutrition 2001, 85(Suppl 2):S193-197.

12. Le HT, Brouwer ID, Verhoef H, Nguyen KC, Kok FJ: Anemia and intestinal parasite infection in school children in rural Vietnam. Asia Pacific Journal of Clinical Nutrition 2007, 16(4):716-723.

13. Lawless JW, Latham MC, Stephenson LS, Kinoti SN, Pertet AM: Iron supplementation improves appetite and growth in anemic Kenyan primary school children. The Journal of nutrition 1994, 124(5):645.

14. Black RE: Zinc deficiency, infectious disease and mortality in the developing world. Journal of Nutrition 2003, 133(5 Suppl 1):1485S-1489S.
15. Thurnham DI: Micronutrients and immune function: some recent developments. Journal of clinical pathology 1997, 50(11):887-891.

16. Best C, Neufingerl N, Del Rosso JM, Transler C, van den Briel T, Osendarp S: Can multi micronutrient food fortification improve the micronutrient status, growth, health, and cognition of schoolchildren? A systematic review. Nutrition Reviews 2011, 69(4):186-204.

17. Bhutta ZA, Ahmed T, Black RE, Cousens S, Dewey K, Giugliani E, Haider BA, Kirkwood B, Morris SS, Sachdev HPS: What works? Interventions for maternal and child undernutrition and survival. Lancet 2008, 371(9610):417-440.

18. Dewey KG, Yang Z, Boy E: Systematic review and meta analysis of home fortification of complementary foods. Maternal Child Nutrition 2009, 5(4):283-321.

19. Serdula M: Maximizing the impact of flour fortification to improve vitamin and mineral nutrition in populations. Food and nutrition bulletin 2010, 31(1 Suppl):S86-93.

20. De-Regil LM, Suchdev PS, Vist GE, Walleser S, Peña-Rosas JP: Home fortification of foods with multiple micronutrient powders for health and nutrition in children under two years of age. Cochrane Database Syst Rev 2011, , 9: CD008959.

21. Walker N, Fischer-Walker C, Bryce J, Bahl R, Cousens S: Standards for CHERG reviews of intervention effects on child survival. International journal of epidemiology 2010, 39(suppl 1):i21-i31.

22. Adu-Afarwuah S, Lartey A, Brown KH, Zlotkin S, Briend A, Dewey KG: Randomized comparison of 3 types of micronutrient supplements for home fortification of complementary foods in Ghana: effects on growth and motor development. American Journal of Clinical Nutrition 2007, 86(2):412-420.

23. Adu-Afarwuah S, Lartey A, Brown KH, Zlotkin S, Briend A, Dewey KG: Home fortification of complementary foods with micronutrient supplements is well accepted and has positive effects on infant iron status in Ghana. American Journal of Clinical Nutrition 2008, 87(4):929-938.

24. Agostoni C, Riva E, Giovannini M: Functional ingredients in the complementary feeding period and long-term effects. Nestle Nutrition Workshop Series Paediatric Programme 2007, 60:123-135, discussion 135-128.

25. Kounnavong S, Sunahara T, Mascie-Taylor CG, Hashizume M, Okumura J, Moji K, Boupha B, Yamamoto T: Effect of daily versus weekly home fortification with multiple micronutrient powder on haemoglobin concentration of young children in a rural area, Lao People's Democratic Republic: a randomised trial. Nutrition Journal 2011, 10:129.

26. Kumar MV, Rajagopalan S: Multiple micronutrient fortification of salt and its effect on cognition in Chennai school children. Asia Pacific Journal of Clinical Nutrition 2007, 16(3):505-511.

27. Lundeen E, Schueth T, Toktobaev N, Zlotkin S, Hyder SM, Houser R: Daily use of Sprinkles micronutrient powder for 2 months reduces anemia among children 6 to 36 months of age in the Kyrgyz Republic: a clusterrandomized trial. Food \& Nutrition Bulletin 2010, 31(3):446-460.

28. Macharia-Mutie CW, Moretti $D$, Van den Briel N, Omusundi AM, Mwangi AM, Kok FJ, Zimmermann MB, Brouwer ID: Maize porridge enriched with a micronutrient powder containing low-dose iron as NaFeEDTA but not amaranth grain flour reduces anemia and iron deficiency in Kenyan preschool children. Journal of Nutrition 2012, 142(9):1756-1763.

29. Menon P, Ruel MT, Loechl CU, Arimond M, Habicht JP, Pelto G, Michaud L: Micronutrient Sprinkles reduce anemia among 9- to 24-mo-old children when delivered through an integrated health and nutrition program in rural Haiti. Journal of Nutrition 2007, 137(4):1023-1030.

30. Osei AK, Rosenberg $\mathrm{HH}$, Houser RF, Bulusu S, Mathews M, Hamer DH: Community-level micronutrient fortification of school lunch meals improved vitamin A, folate, and iron status of schoolchildren in Himalayan villages of India. Journal of Nutrition 2010, 140(6):1146-1154

31. Sharieff W, Yin SA, Wu M, Yang Q, Schauer C, Tomlinson G, Zlotkin S: Shortterm daily or weekly administration of micronutrient Sprinkles has high compliance and does not cause iron overload in Chinese schoolchildren: a cluster-randomised trial. Public Health Nutrition 2006, 9(3):336-344.

32. Varma JL, Das S, Sankar R, Mannar MG, Levinson FJ, Hamer DH: Community-level micronutrient fortification of a food supplement in India: a controlled trial in preschool children aged 36-66 mo. American Journal of Clinical Nutrition 2007, 85(4):1127-1133.

33. Giovannini M, Sala D, Usuelli M, Livio L, Francescato G, Braga M, Radaelli G, Riva E: Double-blind, placebo-controlled trial comparing effects of 
supplementation with two different combinations of micronutrients delivered as sprinkles on growth, anemia, and iron deficiency in Cambodian infants. Journal of pediatric gastroenterology and nutrition 2006, 42(3):306-312.

34. Sharieff W, Yin S, Wu M, Yang Q, Schauer C, Tomlinson G, Zlotkin S: Shortterm daily or weekly administration of micronutrient Sprinkles has high compliance and does not cause iron overload in Chinese schoolchildren: a cluster-randomised trial. Public Health Nutr 2006, 9(3):336-344.

35. Suchdev PS, Ruth L, Obure A, Were V, Ochieng C, Ogange L, Owuor M, Ngure F, Quick R, Juliao P: Monitoring the marketing, distribution, and use of Sprinkles micronutrient powders in rural western Kenya. Food \& Nutrition Bulletin 2010, 31(Supplement 2):168-178.

36. Suchdev PS, Ruth $L$, Woodruff BA, Mbakaya C, Mandava U, Flores-Ayala R, Jefferds MED, Quick R: Selling Sprinkles micronutrient powder reduces anemia, iron deficiency, and vitamin A deficiency in young children in Western Kenya: a cluster-randomized controlled trial. The American journal of clinical nutrition 2012, 95(5):1223-1230.

37. Jack SJ, Ou K, Chea M: Effect of micronutrient Sprinkles on reducing anemia: a cluster-randomized effectiveness trial. Arch Pediatr Adolesc Med 2012, 166(9):842-850

38. Soofi S, Cousens S, Iqbal SP, Akhund T, Ahmed I, Zaidi AKM, Bhutta ZA: Effect of provision of daily zinc and iron with several micronutrients on growth and morbidity among young children in Pakistan: a clusterrandomised trial. Lancet 2013, 382(9886):29-40.

39. Vinod Kumar M, Rajagopalan S: Trial using multiple micronutrient food supplement and its effect on cognition. Indian Journal of Pediatrics 2008, 75(7):671-678

40. Gera T, Sachdev HPS: Effect of iron supplementation on incidence of infectious illness in children: systematic review. BMJ 2002, 325(7373):1142

doi:10.1186/1471-2458-13-S3-S22

Cite this article as: Salam et al:: Effectiveness of Micronutrient Powders (MNP) in women and children. BMC Public Health 2013 13(Suppl 3):S22.

\section{Submit your next manuscript to BioMed Central and take full advantage of:}

- Convenient online submission

- Thorough peer review

- No space constraints or color figure charges

- Immediate publication on acceptance

- Inclusion in PubMed, CAS, Scopus and Google Scholar

- Research which is freely available for redistribution

Submit your manuscript at www.biomedcentral.com/submit 